(6)

OPEN ACCESS

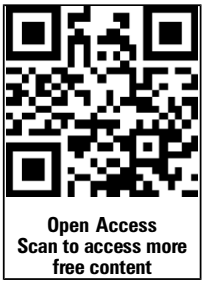

- Additional material is published online only. To view please visit the journal online (http://dx.doi.org/10.1136/ jnnp-2013-307478)

${ }^{1}$ Division of Applied Medicine, Department of Medicine and Therapeutics, Polwarth Building, Foresterhill, University of Aberdeen, Aberdeen, UK ${ }^{2}$ Stroke Unit, Monklands General Hospital, Monkscourt Avenue, Airdrie, UK

${ }^{3}$ Information Services Division, NHS National Services Scotland, Edinburgh, UK ${ }^{4}$ Division of Clinical Neurosciences, University of Edinburgh, Western General Hospital, Edinburgh, UK

${ }^{5}$ Academic Section of Geriatric Medicine, University of Glasgow, Royal Infirmary, Glasgow, UK

${ }^{6}$ Division of Applied Medicine, Department of Medicine and Therapeutics, Polwarth Building, Foresterhill, University of Aberdeen, Aberdeen, UK

Correspondence to Dr Mary Joan Macleod, Division of Applied Medicine, Department of Medicine and Therapeutics, Polwarth Building, Foresterhill, University of Aberdeen, Aberdeen AB25 2ZD, UK; m.j.macleod@abdn.ac.uk

Received 7 January 2014 Revised 8 May 2014 Accepted 27 May 2014 Published Online First 25 June 2014

\section{CrossMark}

\section{To cite: Turner $\mathrm{M}$} Barber M, Dodds $\mathrm{H}$, et al.

J Neurol Neurosurg

Psychiatry 2015;86:

314-318.

\title{
The impact of stroke unit care on outcome in a Scottish stroke population, taking into account case mix and selection bias
}

\author{
Melanie Turner, ${ }^{1}$ Mark Barber, ${ }^{2}$ Hazel Dodds, ${ }^{3}$ Martin Dennis, ${ }^{4}$ Peter Langhorne, ${ }^{5}$ \\ Mary Joan Macleod, ${ }^{6}$ on behalf of the Scottish Stroke Care Audit
}

\begin{abstract}
Background and aim Randomised trials indicate that stroke unit care reduces morbidity and mortality after stroke. Similar results have been seen in observational studies but many have not corrected for selection bias or independent predictors of outcome. We evaluated the effect of stroke unit compared with general ward care on outcomes after stroke in Scotland, adjusting for case mix by incorporating the six simple variables (SSV) model, also taking into account selection bias and stroke subtype.
\end{abstract}

Methods We used routine data from National Scottish datasets for acute stroke patients admitted between 2005 and 2011. Patients who died within 3 days of admission were excluded from analysis. The main outcome measures were survival and discharge home. Multivariable logistic regression was used to estimate the OR for survival, and adjustment was made for the effect of the SSV model and for early mortality. Cox proportional hazards model was used to estimate the hazard of death within 365 days.

Results There were 41692 index stroke events; 79\% were admitted to a stroke unit at some point during their hospital stay and $21 \%$ were cared for in a general ward. Using the SSV model, we obtained a receiver operated curve of 0.82 (SE 0.002) for mortality at 6 months. The adjusted OR for survival at 7 days was $3.11(95 \% \mathrm{Cl} 2.71$ to 3.56$)$ and at 1 year $1.43(95 \% \mathrm{Cl}$ 1.34 to 1.54 ) while the adjusted OR for being discharged home was $1.19(95 \% \mathrm{Cl} 1.11$ to 1.28$)$ for stroke unit care.

Conclusions In routine practice, stroke unit admission is associated with a greater likelihood of discharge home and with lower mortality up to 1 year, after correcting for known independent predictors of outcome, and excluding early non-modifiable mortality.

\section{INTRODUCTION}

Meta-analyses of randomised control trials indicate that stroke unit care (defined by the Stroke Unit Trialists' Collaboration as care of the patient in a defined area with specialist multidisciplinary staffing) is effective in reducing death, dependency and increasing likelihood of return to home in patients with acute stroke. ${ }^{1}$ Stroke units are now recommended as the minimum standard of care for all patients admitted to hospital with a diagnosis of stroke and in Scotland there are specific recommendations that patients should be admitted to a stroke unit and remain there until their inpatient stroke specific care is complete. ${ }^{2}$ Stroke unit care has been shown to be both clinically effective and cost effective, with the main gains in years of life saved. ${ }^{3}$ Data on the advantages of stroke unit admission in the 'real clinical setting' are more limited but also confirm better long-term survival in various patient subgroups. $^{4-7}$

One of the difficulties in interpreting observational data relating stroke unit care to outcomes in the non-trials situation is possible selection bias for stroke unit admissions, especially as the proportion of patients admitted to specialist care varies considerably across Europe. ${ }^{8}$ Deaths occurring early after admission are likely to be influenced by nonmodifiable factors such as stroke severity, age and prestroke disability, ${ }^{9}$ and this might bias the likelihood of admission to a stroke unit bed. For example, if there is a delay in entry to a stroke unit, those who die very early will not access stroke unit care, and this may bias the stroke unit group to those with better early outcomes.

Prediction models for long-term outcome after stroke have been developed to adjust for important case mix variables. These have used clinical features alone or in combination with radiological-derived variables. $^{10-15}$ The six simple variables (SSV) include age at admission, prestroke living arrangement, prestroke independence, arm power at admission, ability to walk at admission and normal verbal component of the Glasgow Coma Scale at admission. This model has been shown to perform as well as or better than other simple predictive systems for predicting the outcome of being alive and independent at 6 months and 1 year after stroke. ${ }^{10}$ The addition of CT-derived variables does not add prognostic power to simple clinical variables. ${ }^{15}$ A systematic review of case mix adjustment models for stroke identified that the SSV model demonstrated statistical robustness, good discriminatory function in external validation studies and comprises variables that are clinically feasible to collect at ward level by non-specialist staff. ${ }^{16}$ The use of the SSV model in comparing outcome in patients admitted or not admitted to stroke units has not been previously investigated.

The Scottish Stroke Care Audit (SSCA) collects information about stroke care in all hospitals managing acute stroke in Scotland. Data collection started in 2002 and has covered all hospitals in Scotland since 2005. The Information Services Division (ISD) of National Health Service (NHS) 
Scotland also collects routine data on hospital admissions, diagnoses and mortality. These comprehensive datasets give the opportunity to link admission to hospital with stroke to subsequent outcomes.

The aim of this study is to evaluate the effect of stroke unit care compared with general ward care on outcomes (mortality, discharge home) after stroke, taking into account case mix variation, selection bias and stroke subtype in a Scotland-wide population.

\section{METHODS}

\section{Data sources}

We obtained data from ISD of NHS National Services Scotland and the General Register Office (GRO) for Scotland.

The SSCA covers 36 acute hospitals across Scotland that admit patients with stroke, usually according to postcode. Twenty-three hospitals had a designated stroke unit prior to January 2005. Of the rest, seven opened a designated stroke unit during 2005, three during 2009 and one new hospital opened in 2011 replacing two hospitals which had stroke units since 2005. Those who opened stroke units later were small district general hospitals, and the numbers of patients affected is relatively small (1002, 2.4\% patients). Two small island boards transferred most patients to another hospital stroke unit for early management before transfer back to a stroke rehabilitation bed. Information was obtained for all stroke patients admitted between 1 January 2005 and 15 September 2011 at all of these acute hospitals.

The GRO records information relating to all deaths, including cause of death, in Scotland. The use of a unique patient identifier, the Community Health Index number, allows all records from SSCA and the GRO death registry to be linked. Linkage was carried out by ISD Scotland and then pseudo-anonymised prior to release to our research group for data analysis.

\section{Data variables}

We included all index stroke events, defined as stroke at final discharge diagnosis and occurring between 1 January 2005 and 15 September 2011. We classified patients as either survival/no survival by 7, 30, 60, 90 and 365 days after hospital admission or after stroke occurrence if already hospitalised. Length of stay was defined as the time from hospital admission or stroke occurrence if already hospitalised, until death or discharge from hospital. Discharge destination in the dataset includes discharged home or to usual place of residence, to another acute hospital, care home, NHS continuing care, an over-riding diagnosis, death, rehabilitation and other.

We investigated the benefits of stroke unit care and how it affects outcomes and the impact of short-term and long-term survival of patients at $7,30,60,90$ and 365 days following admission. The main outcome measure was survival and the secondary outcome was discharge to home/usual place of residence.

\section{Controlling for bias}

Patients who died on the day of admission to hospital and those who were discharged on day of admission were removed from the dataset prior to initial demographic analysis, as it is unlikely that the type of ward care they received would impact on the measured outcomes.

Early deaths after stroke may be non-modifiable, ${ }^{9}$ and may result in the patient dying before accessing a stroke unit bed or being deemed too ill to benefit from a stroke unit environment. To reduce this potential source of bias, patients who died less than and including 3 days postadmission were then removed from the dataset before models were fitted.

\section{Statistical methods}

We performed data management and statistical analyses using SPSS V.20 and SAS V.9.2. Data are shown using standard descriptive statistics. Baseline patient characteristics in the different care groups were compared using $\chi^{2}$ test for categorical and ANOVA for continuous variables.

The SSV model was validated for mortality on the dataset by logistic regression analysis using backward selection methods and calculating the area under a receiver operating characteristic (ROC) curve of sensitivity versus 1 minus specificity. An area of 1 implies a test with perfect sensitivity and specificity, while an area of 0.5 implies that the model's predictions are no better than chance.

We used logistic regression to estimate the OR for survival at 7, 30, 60, 90 and 365 days postadmission. Multivariable logistic regression models were fitted to the data set to adjust the effect of stroke unit care for the SSV (age in years at time of admission, living alone, independent in activities of daily living, able to lift both arms at first assessment, able to talk, able to walk). Age was a continuous variable, while the others were categorical. Other predictor variables significant on univariate analysis were also investigated, including sex, social deprivation (based on postcode ${ }^{17}$ ), presence of atrial fibrillation and type of stroke (ischaemic vs haemorrhagic). Interaction terms were also considered among sex, type of stroke (ischaemic vs haemorrhagic) and the SSV. Logistic regression for the outcome of survival at 365 days post admission for stroke was also analysed according to the delay in admission to a stroke unit following hospital admission. Adjustment for the SSV and type of stroke was carried out.

We used Cox proportional hazards model to estimate the hazard of death within 365 days of admission, adjusting for the SSV, plotting Kaplan-Meier curves, performing a log rank test and calculating hazard ratios (HRs). The outcome of discharged to home was also investigated using logistic regression adjusting for the effects of the SSV and type of stroke to estimate the effect of stroke unit care.

\section{RESULTS}

There were 41692 index stroke events recorded in Scotland between 1 January 2005 and 15 September 2011, after excluding those who died $(n=633)$ or were discharged $(n=1227)$ the same day.

A total of $33074(79 \%)$ stayed in a stroke unit at some point during their hospital stay and 8618 (21\%) were cared for in a general ward. Of those admitted to a stroke unit, 23582 $(71.3 \%)$ were admitted either on the day of admission (day 0 ) or within 1 day (day 1) following their arrival in hospital or after stroke occurring in hospital.

Baseline characteristics for patients treated in stroke units and general wards are shown in table 1 .

There were no differences in the percentage of men admitted to a stroke unit versus general unit. A higher percentage of patients with a high deprivation score accessed stroke unit care compared with general ward care. There was no difference in the percentages accessing stroke unit versus general ward for those with a low deprivation score. For the SSV, fewer patients admitted to stroke unit care were able to lift both their arms or walk, but they were more likely to have been previously independent. In all, $88 \%$ of patients admitted to hospital had a 
Table 1 Baseline characteristics of patients according to type of care

\begin{tabular}{lllc}
\hline Variable & $\begin{array}{l}\text { General ward } \\
(\mathbf{n}=8618)\end{array}$ & $\begin{array}{l}\text { Stroke unit } \\
(\mathbf{n}=33 \text { 074) }\end{array}$ & p value \\
\hline Age, years (mean/median) & $73.6 / 75.8$ & $73.3 / 75.5$ & 0.11 \\
Male gender (\%) & $4180(48.5)$ & $15958(48.2)$ & 0.67 \\
Living alone (\%) & $3066(35.6)$ & $11877(35.9)$ & 0.57 \\
$\begin{array}{l}\text { Current atrial fibrillation at } \\
\text { admission (\%) }\end{array}$ & $1579(18.3)$ & $7326(22.2)$ & $<0.0001$ \\
Independent preadmission (\%) & $6598(76.6)$ & $25736(77.8)$ & 0.01 \\
Lift both arms (\%) & $5317(61.7)$ & $19274(58.3)$ & $<0.001$ \\
Can walk (\%) & $3924(45.5)$ & $11357(34.3)$ & $<0.0001$ \\
Orientated (\%) & $5003(58.1)$ & $19138(57.9)$ & 0.75 \\
Can talk (\%) & $6071(70.4)$ & $23390(70.7)$ & 0.62 \\
Most deprived (\%) & $1437(16.7)$ & $7892(23.9)$ & $<0.0001$ \\
Least deprived (\%) & $1147(13.3)$ & $4493(13.6)$ & 0.51 \\
Length of stay, days & $15.5 / 6$ & $33.3 / 15$ & $<0.0001$ \\
(mean/median) & & & \\
Admitted to stroke unit & - & $23582(71.3)$ & - \\
0-1 days postadmission (\%) & & & \\
Ischaemic stroke (\%) & $7395(85.8)$ & $29478(89.1)$ & $<0.0001$ \\
\hline
\end{tabular}

diagnosis of ischaemic stroke, and $12 \%$ had a confirmed haemorrhagic stroke (table 2).

The numbers and percentages of patients who survived after 7, 30, 60, 90 and 365 days following admission for stroke are shown in table 3 . Patients were more likely to have survived at all time points if they had been admitted to a stroke unit. Table 3 also shows the adjusted ORs for the outcome of survival at five different time points according to stroke unit care versus general ward care.

All variables were significant for the outcome of survival at 6 months, apart from living alone. A ROC of 0.82 (SE 0.002) for mortality at 6 months was calculated (seen in the graph in online supplementary web appendix). The adjusted OR for survival at 7 days post admission for stroke unit care was 3.11 (95\% CI 2.71 to 3.56 ). By 1 year following admission, this decreased to 1.43 (95\% CI 1.34 to 1.54$)$.
In the logistic regression analyses, the addition of other significant predictor variables, sex, social deprivation, year of admission, presence of atrial fibrillation and type of stroke did not significantly alter the ORs seen with only the SSV variables. The effect of adding in stroke type on the OR of survival is shown in table 4.

We also investigated interaction terms. A significant interaction existed between being independent and living alone preadmission for survival at 30,60,90 and 365 days. The OR for survival at 365 days for those admitted to a stroke unit was 1.42 (95\% CI 1.32 to 1.52 ) after adjustment for the interaction term between being independent and living alone preadmission.

The adjusted OR for being discharged home/usual place of residence at 6 months for those admitted to stroke unit care was 1.19 (95\% CI 1.11 to 1.28$)$.

The Kaplan-Meier curves (figure 1) show that for survival to 365 days post admission after adjustment for SSV and type of stroke, stroke unit care has a higher survival distribution compared with general ward care.

The ORs of survival at 365 days following admission according to time to stroke unit admission are shown in table 5 .

Following adjustment, the OR of survival is less for those admitted to a stroke unit after days 0-1 following hospital admission.

\section{DISCUSSION}

Based on evidence from randomised trials, the value of stroke unit admission is now accepted and reflected in many national and international guidelines. However, there remain concerns about how well stroke unit care is implemented in routine practice. ${ }^{18}$ This observational study of practice within a whole country found a significant increase in the odds of survival up to 1 year after admission and of discharge to home/usual place of residence for patients admitted to a stroke unit compared with those cared for in a general ward. Previous observational studies have mainly focused on short-term to mid-term outcomes with only a few investigating death up to 1 year or beyond. ${ }^{5-7} 18-21$ Data from registries confirming the benefits of stroke unit care have not necessarily corrected for potential selection bias or known independent predictors of outcome, and some predate comprehensive secondary prevention guidelines. ${ }^{21}$ The Austrian

Table 2 Baseline characteristics of patients according to type of stroke and type of care

\begin{tabular}{|c|c|c|c|c|}
\hline \multirow[b]{2}{*}{ Variable } & \multicolumn{2}{|c|}{ Ischaemic $(n=36873 / \%=88.4)$} & \multicolumn{2}{|c|}{ Haemorrhagic $(n=4819 / \%=11.6)$} \\
\hline & $\begin{array}{l}\text { Stroke unit } \\
(\mathrm{n}=29478)\end{array}$ & $\begin{array}{l}\text { General ward } \\
(\mathrm{n}=7395)\end{array}$ & $\begin{array}{l}\text { Stroke unit } \\
(\mathrm{n}=3596)\end{array}$ & $\begin{array}{l}\text { General ward } \\
(n=1223)\end{array}$ \\
\hline Age, years (mean/median) & 73.3/75.4 & $73.5 / 75.7$ & $73.8 / 76.1$ & $74.1 / 76.6$ \\
\hline Male gender (\%) & $14216(48.2)$ & $3634(49.1)$ & $1742(48.4)$ & $546(44.6)$ \\
\hline Living alone (\%) & $10601(36.0)$ & $2645(35.8)$ & $1276(35.5)$ & $421(34.4)$ \\
\hline Current atrial fibrillation at admission (\%) & $6745(22.9)$ & 1399 (18.9) & $581(16.2)$ & $180(14.7)$ \\
\hline Independent preadmission (\%) & $22977(77.9)$ & $5708(77.2)$ & $2759(76.7)$ & $890(72.8)$ \\
\hline Lift both arms (\%) & $17741(60.2)$ & $4908(66.4)$ & $1533(42.6)$ & 409 (33.4) \\
\hline Can walk (\%) & $10524(35.7)$ & $3663(49.5)$ & $833(23.2)$ & $261(21.3)$ \\
\hline Orientated (\%) & $17561(59.6)$ & $4641(62.8)$ & $1577(43.9)$ & 362 (29.6) \\
\hline Can talk (\%) & $21224(72.0)$ & $5554(75.1)$ & $2166(60.2)$ & $517(42.3)$ \\
\hline Most deprived (\%) & $7167(24.3)$ & $1271(17.2)$ & $725(20.2)$ & $166(13.6)$ \\
\hline Least deprived (\%) & $3891(13.2)$ & $971(13.1)$ & $602(16.7)$ & $176(14.4)$ \\
\hline Length of stay, days (mean/median) & $32.2 / 14.0$ & $15.7 / 6.0$ & $42.7 / 23.0$ & $13.8 / 5.0$ \\
\hline $\begin{array}{l}\text { Of those admitted to stroke unit, admitted within } 0-1 \text { days post } \\
\text { admission (\%) }\end{array}$ & 21137 (71.7) & & $2445(68.0)$ & \\
\hline
\end{tabular}


Table 3 Numbers of patients and adjusted (for SSV) ORs for outcome of survival post admission (cumulative) according to type of care

\begin{tabular}{lllll}
\hline $\begin{array}{l}\text { Survival post } \\
\text { admission } \\
\text { (days) }\end{array}$ & $\begin{array}{l}\text { General } \\
\text { ward } \\
\mathbf{n = 7 8 1 6} \\
(\%)\end{array}$ & $\begin{array}{l}\text { Stroke unit } \\
\mathbf{n = 3 2 ~ 4 5 9} \\
(\%)\end{array}$ & $\begin{array}{l}\text { Adjusted OR } \\
\text { for stay in } \\
\text { stroke unit* }\end{array}$ & $\mathbf{9 5 \% ~ C l}$ \\
\hline 7 & $7364(94.2)$ & $31635(97.5)$ & 3.11 & 2.71 to 3.56 \\
30 & $6621(84.7)$ & $29258(90.4)$ & 2.22 & 2.03 to 2.43 \\
60 & $6319(80.9)$ & $28004(86.3)$ & 1.94 & 1.79 to 2.10 \\
90 & $6166(78.9)$ & $27202(83.8)$ & 1.76 & 1.63 to 1.90 \\
365 & $5535(70.8)$ & $24137(74.4)$ & 1.43 & 1.34 to 1.54 \\
\hline *Adjusted for age in years, living alone, independent in activities of daily living, able \\
to lift both arms, able to walk and able to talk at admission. \\
SSV, six simple variables.
\end{tabular}

and Berlin stroke registries confirm that age is the strongest predictor of outcome after stroke admission. ${ }^{92}$ We have shown that even after removing patients with early mortality from the data and correcting for known predictors of outcome, stroke unit care remains associated with improved outcome after admission with a stroke. While not directly measuring the impact of dementia or other comorbidities which might bias stroke unit admission, the SSV does take into account prior independence and whether the patient was living alone.

In acute stroke patients, up to $70 \%$ of early death and poor outcome is attributable to non-modifiable factors, including age, stroke severity and prestroke disability. ${ }^{9}$ These parameters are reflected in the SSV model, but are also likely to cause selection bias for admission to stroke unit beds. In an attempt to further correct for these non-modifiable predictors of outcome which might also have influenced stroke unit admission, we removed from the analyses all patients who died within 3 days of admission. The benefit of accessing a stroke unit was still present. The factors which influence mortality and outcome after the first few days of admission, such as DVT, aspiration pneumonia and cardiac complications, are amenable to prevention and treatment, and thus more likely to be influenced by specialist stroke unit care. For patients who are unable to access stroke unit care, the NHS in Scotland provides guidelines for standards of care in acute general medical wards. ${ }^{23}$ The main differences from stroke units are in education and training: nurses in stroke units are required to undertake stroke specific education, for example, on swallow screening and physiological monitoring. In all, 23 of 36 hospitals had an established acute stroke unit before 2005, an additional seven opened a unit during 2005 and all had a

Table 4 ORs for outcome of survival post admission (cumulative) according to type of care (adjusted for SSV and type of stroke)

\begin{tabular}{lll}
\hline $\begin{array}{l}\text { Survival post admission } \\
\text { (days) }\end{array}$ & $\begin{array}{l}\text { Adjusted OR for stay in stroke } \\
\text { unit* }\end{array}$ & $\mathbf{9 5 \%} \mathrm{Cl}$ \\
\hline 7 & 3.03 & 2.64 to 3.48 \\
30 & 2.20 & 2.01 to 2.40 \\
60 & 1.92 & 1.77 to 2.09 \\
90 & 1.75 & 1.62 to 1.89 \\
365 & 1.43 & 1.33 to 1.53 \\
\hline *Adjusted for age in years, living alone, independent in activities of daily living, able \\
to lift both arms, able to walk, able to talk at admission and type of stroke \\
(ischaemic vs haemorrhagic).
\end{tabular}

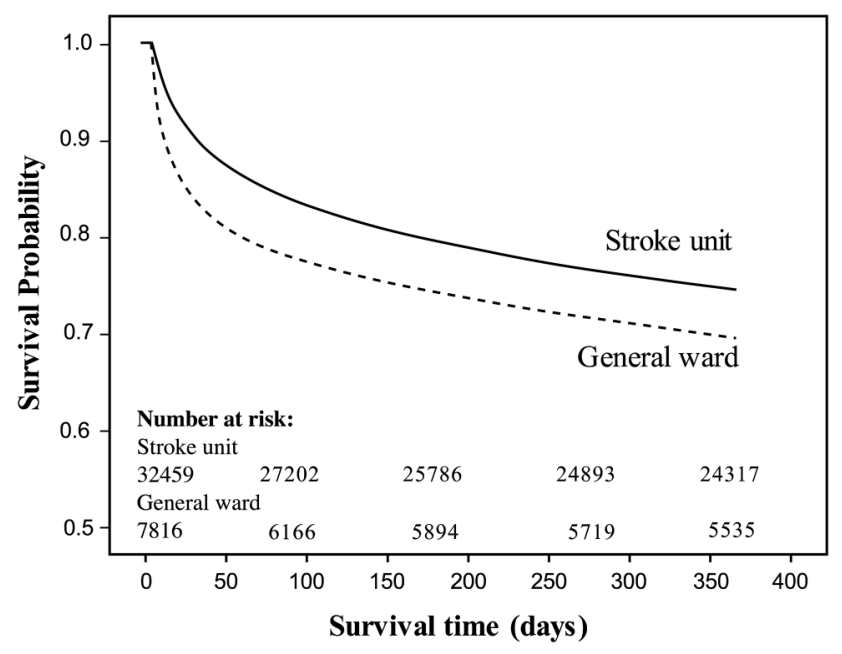

Figure 1 Survival curve according to admission to general ward or stroke unit.

functioning stroke unit by 2010. Those who opened stroke units later were small district general hospitals, and the numbers of patients affected are relatively small.

Some hospitals may be more selective for stroke unit admissions than others, taking into account additional comorbidities at admission. This could introduce further bias on outcomes. Admission to a stroke unit is also dependent upon the availability of a bed at the time of a patient's admission. Age affected likelihood of stroke unit admission, possibly reflecting the poorer health of older patients. Differences in outcomes could therefore also be dependent on some unmeasured variables. Deprivation did not appear to adversely affect admissions: this might reflect the location of stroke units in areas with larger relatively deprived populations such as the central belt of Scotland. This is different from Canadian data which suggested patients from lower socio-economic classes were less likely to be admitted to a stroke unit. $^{22}$

American, ${ }^{24}$ European $^{25}$ and UK guidelines ${ }^{26}$ recommend immediate or early stroke unit care. Survival to 1 year showed a significant benefit from early admission, unadjusted and adjusted for the SSV, perhaps due to better management of modifiable risk factors. Although the scale of benefit falls with time after the index event, probably due to recurrent events and the progression of comorbidities, survival to 1 year better reflects the 'effect' of stroke unit care and the benefit of early admission.

We found the SSV model to be simple to use and relatively easy to collect the required variables in a large population. ${ }^{27} \mathrm{~A}$ recent systematic review of case mix adjustment models for stroke confirms its external validity. ${ }^{16}$ Not surprisingly, living

Table 5 OR of survival at 365 days after admission by timing of stroke unit admission (adjusted for case mix using SSV and type of stroke)

\begin{tabular}{lc}
\hline Admission to stroke unit (time in days) & $\begin{array}{c}\text { Survival at } 365 \text { days } \\
\text { OR }(95 \% \mathrm{Cl})\end{array}$ \\
\hline $0-1$ & 1 \\
2 & $0.89(0.80$ to 0.99$)$ \\
3 & $0.88(0.76$ to 1.01$)$ \\
$\geq 4$ & $0.78(0.71$ to 0.86$)$ \\
\hline SSV, six simple variables. &
\end{tabular}


alone is not predictive of death, and in some studies does not predict independent survival. ${ }^{27}$ In this study, living alone did not predict survival at 6 months or discharge home, although this variable was originally incorporated in the model to predict whether someone would be alive and living at home at 6 months and 1 year. We calculated a ROC of 0.82 (SE 0.002) for mortality at 6 months compared with a ROC of 0.85 (SE 0.01) in the original SSV study. ${ }^{10}$ The other model variables provided good predictive value. We confirm that the five simple variables are robust in a large routinely collected dataset.

The main strengths of our study are its size $(n=41692)$, representation of a whole country, capture of case mix adjustors and potential to control for admission bias. The main limitation of this study was that we could only assess the impact of predictors which were measured. Patients who died shortly after admission might have been missed from the audit. No data were available on cognitive decline (likely to be important for stroke unit admission), other than the surrogate measure of orientated at time of admission.

Within Scotland, there are some variations in the levels of social care available in the community, but these apply to patients both in stroke and general wards. In other healthcare settings, local health and social services support may vary and thus the proportion of patients discharged home may be different, but stroke unit care will be relatively independent of these factors. Thus, we believe our results are generalisable.

In summary, stroke unit admission is associated with lower mortality and increases the likelihood of discharge home, even when data are corrected for known independent predictors of outcome, and when early non-modifiable mortality is taken into account. There was benefit of earlier stroke unit admission up to 1 year after stroke.

Acknowledgements We are grateful to David Murphy of the SSCA for providing data and to Lynsey Waugh of ISD Scotland for linking the SSCA data with General Register Office data. We also acknowledge the help of all who enter data into SSCA.

Contributors All authors designed the study. MT performed data analysis, MT and MJM wrote initial draft and all authors commented on the manuscript.

Funding This study was funded by Chest, Heart and Stroke Scotland (Grant no R11/A134). The SSCA is funded by NHS Scotland via ISD. Neither funder had any role in the analysis.

\section{Competing interests None.}

Ethics approval The study was approved by Scotland A Research Ethics Committee, Ref. No.=10/MRE00/76 and the Privacy Advisory Committee of the ISD, NHS Scotland, Ref 76/11.

Provenance and peer review Not commissioned; externally peer reviewed.

Open Access This is an Open Access article distributed in accordance with the Creative Commons Attribution Non Commercial (CC BY-NC 3.0) license, which permits others to distribute, remix, adapt, build upon this work non-commercially, and license their derivative works on different terms, provided the original work is properly cited and the use is non-commercial. See: http://creativecommons.org/ licenses/by-nc/3.0/

\section{REFERENCES}

1 Stroke Unit Trialists' Collaboration. Organised inpatient (stroke unit) care for stroke. In: Cochrane Library. Issue 1, 2007.

2 NHS Quality Improvement Scotland. Clinical standards for stroke services: care of the patient in the acute setting. Edinburgh, Scotland: NHS Quality Improvement Scotland (NHS QIS), 2009.

3 Te Ao BJ, Brown PM, Feigin VL, et al. Are stroke units cost effective? Evidence from a New Zealand stroke incidence and population-based study. Int J Stroke 2012;7:623-30.

4 Seenan P, Long M, Langhorne P. Stroke units in their natural habitat. Stroke 2007;38:1886-92.

5 Di Carlo A, Lamassa M, Wellwood I, et al. Stroke unit care in clinical practice: an observational study in the Florence center of the European Registers of Stroke (EROS) Project. Eur J Neurol 2011;18:686-94.

6 Terent A, Asplund K, Farahmand B, et al. Stroke unit care revisited: who benefits the most? A cohort study of 105043 patients in riks-stroke, the Swedish Stroke Register. J Neurol Neurosurg Psychiatry 2009;80:881-7.

7 Candelise L, Gattinoni M, Bersano A, et al. Stroke-unit care for acute stroke patients: an observational follow-up study. Lancet 2007;369:299-305.

8 Leys DE, Ringelstein B, Kaste M, et al. Facilities available in European hospitals treating stroke patients. Stroke 2007;38:2985-91.

9 Koennecke H-C, Belz W, Berfelde D, et al. Factors influencing in-hospital mortality and morbidity in patients treated on a stroke unit. Neurology 2011;78:965-72.

10 Counsell C, Dennis M, McDowall M. Predicting functional outcome in acute stroke: comparison of a simple six variable model with other predictive systems and informal clinical prediction. J Neurol Neurosurg Psychiatry 2004;75:401-5.

11 Weir $\mathrm{CJ}$, Bradford AP, Lees KR. The prognostic value of the components of the Glasgow Coma Scale following acute stroke. QJM 2003;96:67-74.

12 Hand PJ, Wardlaw JM, Rivers CS, et al. MR diffusion-weighted imaging and outcome prediction after ischemic stroke. Neurology 2000;66:1159-63.

13 Johnston KC, Wagner DP, Haley EC Jr, et al. Combined clinical and imaging information as an early stroke outcome measure. Stroke 2002;33:466-72.

14 Wardlaw JM, Lewis CS, Dennis MS, et al. Is visible infarction on computed tomography associated with an adverse prognosis in acute ischemic stroke? Stroke 1998:29:1315-19.

15 Reid JM, Gubitz GJ, Dai D, et al. Predicting functional outcome after stroke by modelling baseline clinical and CT variables. Age Ageing 2010;39:360-6.

16 Teale EA, Forster A, Munyombwe T, et al. A systematic review of case-mix adjustment models for stroke. Clin Rehabil 2012;26:771-86.

17 A Scottish Government National Statistics Publication. Scottish Index of Multiple Deprivation 2009: General Report. October 2009.

18 Ayis SA, Coker B, Bhalla A, et al. Variations in acute stroke care and the impact of organised care on survival from a European perspective, The European Registers of Stroke (EROS) investigators. J Neurol Neurosurg Psychiatry 2013:84:604-12.

19 Davenport RJ, Dennis MS, Warlow CP. Effect of correcting outcome data for case mix: an example from stroke medicine. BMJ 1996;312:1503-5.

20 Jorgensen HS, Kammersgaard LP, Houth J, et al. Who benefits from treatment and rehabilitation in a stroke unit? A community based study. Stroke 2000;31:434-9.

21 Rudd AG, Hoffman A, Irwin P, et al. Stroke unit care and outcome: results from the 2001 National Sentinel Audit of Stroke (England, Wales and Northern Ireland). Stroke 2005:36:103-6.

22 Knoflach $M$, Matosevic $B$, Rucker $M$, et al. Functional recovery after ischemic stroke —a matter of age. Neurology 2012;78:279-84.

23 The Scottish Government. The healthcare quality strategy for NHS scotland. Edinburgh: The Scottish Government, 2010.

24 Jauch EC, Saver JL, Adams HP, et al. Guidelines for the early management of patients with acute ischemic stroke: a guideline for healthcare professionals from the American Heart Association/American Stroke Association. Stroke 2013;44:870-947.

25 ESO Executive Committee and the ESO Writing Committee. Guidelines for management of ischaemic stroke and transient ischaemic attack 2008. Cerebrovasc Dis 2008;25:457-507.

26 Intercollegiate Stroke Working Party. National clinical guideline for stroke. 4th edn. London: Royal College of Physicians, 2012.

27 Ayis SA, Coker B, Rudd AG, et al. Predicting independent survival after stroke: a European study for the development and validation of standardised stroke scales and prediction models of outcome. J Neurol Neurosurg Psychiatry 2013;84:288-96. 